\title{
INCENTIVOS PARA ESTUDIAR PEDAGOGÍA: EL CASO DE LA BECA VOCACIÓN DE PROFESOR*
}

\author{
Francisco Claro \\ P. Universidad Católica de Chile
}

Magdalena Bennett

London School of Economics

\author{
Ricardo D. Paredes \\ P. Universidad Católica de Chile \\ Tomás Wilson \\ Vicaría de la Pastoral Social \\ $y$ de los Trabajadores
}

\begin{abstract}
Resumen: A fines del año 2010 se instauró en Chile la beca vocación de profesor (BVP). En este trabajo se analiza el efecto de la BVP en el perfil de los alumnos admitidos a las carreras de pedagogía de las universidades agrupadas en el CRUCh (Consejo de Rectores de Universidades Chilenas),
\end{abstract}

Francisco Claro. Ph. D. en Física, Universidad de Oregon, EE.UU. Profesor titular, Facultad de Educación de la P. Universidad Católica de Chile. Dirección electrónica: fclaro@uc.cl.

Ricardo D. Paredes Ph. D. en Economía, UCla, EE.UU. Profesor de la Escuela de Ingeniería de la P. Universidad Católica de Chile. Dirección electrónica: rparedes@ing.puc.cl.

Magdalena Bennett, Magíster en Ciencias de la Ingeniería, Universidad Católica de Chile. Estudiante del MSc en Políticas Sociales, London School of Economics. Dirección electrónica: mbennet3@uc.cl.

Tomás Wilson. Licenciado en Física, Universidad Católica de Chile. Coordinador de la Escuela Sindical de la Vicaría de la Pastoral Social y de los Trabajadores, Arzobispado de Santiago. Dirección electrónica: twilson@uc.cl.

* Agradecemos a la Vicerrectoría de Investigación de la P. Universidad Católica por el financiamiento del proyecto interdisciplinario VRI 09/2010; al MINEDUC por el uso de las bases de datos. Agradecemos también a María Fernanda Ramírez y al editor y referees de Estudios Públicos por sus comentarios. Ricardo Paredes agradece el financiamiento del Proyecto CEPPE, CIE01Conicyt y al proyecto Fondecyt1110485.

Estudios Públicos, 131 (invierno 2013), 37-59.

ISSN 0716-1115 (impresa) ISSN 0718-3089 (en línea). 
así como la variación de ese efecto en las distintas universidades del CRUCh. Utilizando datos de los procesos de admisión del CRUCh y las bases de datos del SIMCE de segundo medio, se constata que la BVP se ha traducido en el ingreso a las carreras de pedagogía de alumnos con mejores puntajes en las pruebas de selección universitaria (PSU) y mejor desempeño previo en la enseñanza secundaria. Más importante, ese mejor perfil de los alumnos no parece obedecer sólo al hecho de que la beca no está condicionada a la situación económica de los postulantes, sino que se asocia a efectos en un residuo de elementos no medidos por los tests de selección, pudiendo en parte obedecer a "habilidades". Por último, el análisis muestra que las universidades preferidas por una proporción mayor de quienes acceden a la BVP son aquellas que previamente admitían a alumnos con mayores puntajes en la PSU.

Palabras clave: formación de profesores, becas educación superior, incentivos para estudiar pedagogía, beca vocación de profesor.

Clasificación JEL: I23, I28.

Recibido: enero 2013; aceptado: julio 2013.

\section{INCENTIVES TO STUDY PEDAGOGY: THE CASE OF THE TEACHER VOCATIONAL SCHOLARSHIP}

Abstract: A teacher vocational scholarship (BVP) was established in Chile at the end of 2010. This article analyzes the effect of the BVP on the profile of the students admitted to the pedagogy programs at universities that are members of the Council of Chancellors of Chilean Universities (CRUCh) and how that effect has varied in the different CRUCh universities. Using data from the CRUCh admission processes and the databases of the high-school sophomore SIMCE testing, it is confirmed that the outcome of the BVP has been students entering pedagogy programs who have earned better scores in the university selection testing (PSU) and performed better during high school. More importantly, that improvement in the student profile does not seem to be due merely to the fact that the scholarship is not conditioned to the applicant's economic situation, but rather to the effects of residual elements not measured by selection testing. It may be partially due to "skills". Lastly, the analysis shows that the universities preferred by a greater proportion of BVP recipients are universities that previously accepted students with higher PSU scores.

Keywords: Teacher formation, university scholarships, incentives to study pedagogy, teacher vocation scholarship.

Clasification JEL: I23, I28.

Received: January 2013; accepted: July 2013. 


\section{Introducción}

$\mathbf{E}_{\text {xiste amplio consenso en que la calidad de los profesores es }}$ un factor determinante en el aprendizaje de los estudiantes. Una de las prácticas recurrentes de países que han tenido los mejores resultados en educación es establecer altas exigencias al momento del ingreso a la carrera de pedagogía. Países líderes de la OECD reclutan a sus profesores entre el mejor $30 \%$ de cada cohorte anual de egresados de la educación secundaria, notablemente Corea del Sur (mejor 5\%), Finlandia (mejor 10\%) y Singapur (30\%) (Barber y Mourshed, 2008; OECD, 2009).

La situación en países menos desarrollados y en Chile en particular es diferente: los postulantes a las carreras de pedagogía provienen en su mayoría del $50 \%$ con peor puntaje de la prueba de selección universitaria (PSU) (Cabezas y Claro, 2011). Además, hay problemas en los currículos de pedagogía que hacen que las escuelas de pedagogía no puedan revertir las carencias de origen (e.g., Larrondo et al., 2007). Más aún, los egresados de pedagogía que tienen menos formación previa tienden a enseñar en las escuelas que forman a los niños de sectores vulnerables, con lo cual se acentúan los problemas de inequidad que afectan a la educación latinoamericana y, en particular, a la chilena (Meckes y Bascopé, 2010; Cabezas y Claro, 2011; Paredes y Ramírez, 2011; Cabezas et al. 2013).

A raíz de este diagnóstico, el año 2010 un Panel de Expertos convocado por el Ministerio de Educación de Chile recomendó la creación de una beca dirigida a incentivar a los mejores egresados de la educación secundaria a optar por la carrera de pedagogía. En particular, incentivar a aquellos estudiantes de alta capacidad y motivación por la pedagogía, y que por razones de costo de los estudios, rentabilidad futura e imagen de la carrera no considerarían pedagogía entre sus opciones.

La Beca Vocación de Profesor (BVP) comenzó a regir para el proceso de admisión 2011, dirigida a jóvenes que obtuvieran un puntaje igual o mayor que 600 puntos en la prueba de selección universitaria (PSU), promedio de notas de enseñanza media mayor que 6,0 y que postularan como primera preferencia a carreras de pedagogía ${ }^{1}$. A diferencia de otras becas del Estado para estudiantes universitarios, la BVP no se condiciona a la situación socioeconómica del alumno.

${ }^{1}$ En la aplicación de la BVP el año 2011 sólo se consideró la exigencia de 600 o más puntos en la PSU. 
El propósito de este trabajo es tener una primera aproximación del efecto de la BVP en el perfil de los alumnos que ingresan a estudiar pedagogía, y de la distribución de ese efecto entre las distintas universidades del CRUCh.

El trabajo se estructura en tres secciones aparte de esta introducción. La Sección 2 describe la BVP y la información empleada. La Sección 3 presenta la metodología, estima el efecto de la BVP en el perfil de los postulantes a pedagogía y desglosa dicho efecto entre factores sociodemográficos del alumno, efectos no medidos asociables en parte a habilidades y la eventual repercusión en el tipo de universidad beneficiada. En la Sección 4 se concluye.

\section{Antecedentes}

\subsection{La BVP e incentivos}

El Estado de Chile entrega diferentes formas de ayuda a los estudiantes para el financiamiento de su educación superior, tanto en créditos como becas de arancel y becas de mantención. Estas ayudas están dirigidas a los estudiantes de las universidades tradicionales (creadas antes del año 1982), que en su conjunto conforman el Consejo de Rectores de las Universidades Chilenas (CRUCh), y están supeditadas a la obtención de un puntaje mínimo en la PSU (Prueba de Selección Universitaria, una prueba estandarizada nacional) y, como se señaló, a provenir de hogares de los primeros quintiles de ingreso ${ }^{2}$. En el caso de las becas de arancel, ellas están orientadas principalmente a alumnos procedentes de los tres primeros quintiles de ingreso socioeconómico. El crédito subsidiado está dirigido a los quintiles superiores, con exclusión del quinto quintil. Las becas financian el pago de una mensualidad respecto de un arancel de referencia, que para algunas carreras representa una fracción del valor real, puesto que, en parte como consecuencia de las mismas políticas de financiamiento, las mensualidades han experimentado incrementos importantes desde comienzos de los 2000.

Para acceder a la BVP durante el proceso de admisión 2011, el postulante debió cumplir los siguientes requisitos: haber quedado seleccionado en una carrera de pedagogía cuyo puntaje de corte, considerado

${ }^{2}$ Para una descripción del sistema de financiamiento estudiantil, véase Comisión de Financiamiento Estudiantil (2012). 
como el promedio entre la prueba de matemáticas y lenguaje, no fuera menor que 500 puntos, y haber obtenido 600 puntos o más en promedio entre la PSU de matemática y la PSU de lenguaje. Considerando que la media en la PSU es de aproximadamente 500 puntos y que históricamente los puntajes de corte y medios en las carreras de pedagogía han sido sustancialmente menores, ello constituyó un desafío muy importante para los planteles. De hecho, 19 de las 22 universidades que impartían alguna carrera de pedagogía en el año 2010 tenían puntajes de corte bajo los 500 puntos $^{3}$. El año 2011, en cambio, todas las universidades cerraron sobre los 500 puntos.

Respecto de otras becas que otorga el Estado, la BVP innova entonces en al menos cinco sentidos: i) no considera factores socioeconómicos sino exclusivamente el puntaje obtenido en la PSU; ii) paga completamente la matrícula y el arancel; iii) requiere que el postulante manifieste pedagogía como su primera preferencia de carrera; iv) requiere que el puntaje PSU de corte de la universidad que ofrece la carrera sea, como mínimo, 500 puntos, y v) requiere que una vez concluidos sus estudios el ex becario trabaje un período de tres años en un establecimiento municipal o subvencionado (Decreto 337 del Ministerio de Educación de Chile, 2010).

Como ya se señaló, el diseño de la BVP se propuso hacer más atractiva la carrera de pedagogía para los alumnos que tienen mayores puntajes y contribuir a la selección de estudiantes con buenos antecedentes académicos. Ello, a su vez, prestigiaría socialmente a la carrera. Del diseño se pueden desprender distintas hipótesis contrastables empíricamente, siendo la más natural que el diseño de la BVP permite seleccionar relativamente mejores estudiantes. Alvarado, Duarte y Neilson (2012), en un estudio desarrollado en paralelo con el nuestro y en el que emplean la metodología de "regresión discontinua", hallan claros indicios de quiebre en la selección de los alumnos en base del diseño de la BVP. El propósito nuestro es, además de verificar la existencia de efectos en los puntajes de selección, hacer una caracterización de los seleccionados, más allá de lo que está determinado por las pruebas de selección

3 Catorce universidades contaban con puntajes de corte de alrededor de 475 puntos y cinco universidades cerraban con puntajes cercanos a los 450 puntos. 
universitaria. De hecho, un tema especialmente relevante, a partir del diseño de la BVP, es la ausencia de una orientación hacia los jóvenes más vulnerables. La caracterización sociodemográfica es uno de los focos de nuestro estudio. Finalmente, también hacemos un esfuerzo por relacionar en forma primaria el efecto de la BVP en las distintas universidades. En la medida en que la calidad de las universidades es heterogénea, pudiera existir un efecto positivo si la BVP induce a las universidades a mejorar sus deficiencias y a ofrecer mejores programas de pedagogía, así como a seleccionar alumnos con mejores estándares académicos. Antes de la BVP, para decidir a cuál universidad postular, los interesados en pedagogía debían considerar, aparte del puntaje obtenido en la PSU, el hecho presumible de que las mejores universidades cobraban un arancel más alto, y entonces aquellos que obtenían una beca del Estado debían pagar la diferencia entre el arancel real y el de referencia. Con la BVP los postulantes sólo tienen que considerar la calidad de los programas de Educación que ofrece cada plantel, por lo que las mejores universidades resultan más atractivas.

\subsection{Descripción estadística}

Los datos de admisión del conjunto de universidades que conforman el Consejo de Rectores de las Universidades Chilenas (CRUCH) tienen carácter público, a diferencia de los referidos al resto de las universidades creadas con posterioridad al año 1981. Hasta el proceso de admisión del 2011 las universidades del CRUCH se sometían a un sistema único de postulación, en base a la PSU como principal criterio de selección. Por estas dos cualidades, accesibilidad y uniformidad de criterios, en este estudio consideramos la base de datos de las 25 universidades integrantes del $\mathrm{CRUCH}$, entendiendo que el efecto que se visualiza en ellas representa una parte del efecto total. En adelante nos referiremos en forma exclusiva a este conjunto de planteles, que contiene algunas de las instituciones de mayor calidad reconocida y, por cierto, las más antiguas del país.

Integramos dos archivos de datos, uno que contiene los puntajes obtenidos en la PSU y otro que informa acerca de las variables relevantes a la postulación a las diversas carreras que ofrece el sistema de uni- 
versidades agrupadas en el CRUCh. En primer lugar verificamos el perfil de los postulantes hasta el año 2010 comparando diversos procesos. Hallamos que éste fue muy similar en cuanto a nivel socioeconómico y puntaje de postulación, notando variaciones mínimas en el tiempo (véase Wilson, 2011). De este modo, podemos señalar que los postulantes del proceso 2009 son representativos de aquellos "antes de la existencia de la BVP" (período 2005-2010). De esta constatación, para determinar el efecto de la BVP comparamos los datos pertinentes a los procesos de admisión 2009 y 2011, esto es, antes y después de la instauración de la BVP.

Habiendo identificado a los postulantes y seleccionados, procedimos a integrar esta base con información derivada de la prueba SIMCE, una prueba estandarizada de medición de aprendizaje escolar (Sistema de Medición de la Calidad de la Educación) para los años 2006 y 2008. Las cohortes que rindieron la prueba SIMCE esos años corresponden a los alumnos que postularon a las universidades por primera vez los años 2009 y $2011^{4}$. Con ello pudimos incorporar a la base de postulantes información relativa a desempeño escolar y a situación social y demográfica de ellos y sus familias.

Una primera mirada sobre las poblaciones y sus postulaciones está dada por la evolución y recomposición de matrículas y preferencias en el tiempo. La Tabla $\mathrm{N}^{\circ} 1$ muestra la composición de la matrícula de primer año de las universidades del CRUCh, por diferentes áreas en varios años. Allí se observa que la composición porcentual de postulantes aceptados a las diferentes áreas no cambió sustancialmente entre 2007 y 2010, años previos a la BVP. Particularmente la matrícula en el área de Educación se mantuvo muy estable en un $10 \%$ de los jóvenes que rindieron la PSU (y el 15\% si sólo se considera el total de los más de 60 mil seleccionados en diferentes carreras del CRUCh). Sin embargo, la cantidad absoluta de postulantes aceptados en educación disminuyó levemente entre 2010 y 2011, a pesar del incremento absoluto en el número total de aceptados en todas las carreras en el proceso de admisión 2011.

En cuanto a la evolución de los puntajes promedio de la PSU entre 2007 y 2011 (promedio de promedios de pruebas de Matemática y

${ }^{4}$ Del total de egresados de la enseñanza media el año 2008 (proceso PSU 2009), el 18\% (37.005) de la muestra se pierde debido a que no cuentan con datos del SIMCE 2006, mientras que para el proceso 2011 dicho porcentaje es $21 \%$ (45.116). 


\begin{tabular}{lrrrrr}
\hline & \multicolumn{5}{c}{ Proceso de admisión } \\
\cline { 2 - 6 } Área & 2007 & 2008 & 2009 & 2010 & 2011 \\
\hline Educación & $9,9 \%$ & $10,6 \%$ & $9,7 \%$ & $10,5 \%$ & $10,3 \%$ \\
Economía y Leyes & $5,6 \%$ & $5,9 \%$ & $5,2 \%$ & $6,0 \%$ & $6,2 \%$ \\
Medicina y Odontología & $1,6 \%$ & $1,7 \%$ & $1,6 \%$ & $1,7 \%$ & $1,8 \%$ \\
Otras carreras de la salud & $4,9 \%$ & $5,2 \%$ & $4,7 \%$ & $5,3 \%$ & $6,0 \%$ \\
Ciencias & $4,9 \%$ & $5,2 \%$ & $4,6 \%$ & $4,7 \%$ & $4,7 \%$ \\
Agronomía & $4,1 \%$ & $4,0 \%$ & $3,6 \%$ & $3,7 \%$ & $3,5 \%$ \\
Ingeniería & $15,7 \%$ & $17,2 \%$ & $16,5 \%$ & $18,7 \%$ & $19,8 \%$ \\
Ciencias Sociales, Humanidades & $11,8 \%$ & $12,7 \%$ & $11,3 \%$ & $12,4 \%$ & $12,5 \%$ \\
y Servicios & $3,7 \%$ & $3,8 \%$ & $3,5 \%$ & $3,9 \%$ & $3,9 \%$ \\
Artes y Arquitectura & $1,8 \%$ & $1,9 \%$ & $1,7 \%$ & $2,0 \%$ & $2,2 \%$ \\
Bachillerato & $36,0 \%$ & $31,7 \%$ & $37,7 \%$ & $31,0 \%$ & $29,0 \%$ \\
No seleccionado & 61.772 & 62.758 & 64.784 & 67.013 & 67.803 \\
Total seleccionados & 96.547 & 91.862 & 103.929 & 97.150 & 95.536 \\
Total alumnos que rindieron PSU & & & & &
\end{tabular}

Fuente: Elaboración propia en base archivos PSU-DEMRE 2007-2011.

Lenguaje) existen fluctuaciones que son menores que diez puntos en las distintas carreras. La excepción es Educación, que en 2011, año en que se instauró la BVP, aumenta 15 puntos, alcanzando el puntaje promedio del área de Ciencias Sociales y Humanidades. El promedio de Educación está claramente afectado por la eliminación de puntajes PSU bajo 500 en las carreras que se hicieron elegibles para la BVP. Sin embargo, si sólo se consideran aquellos alumnos que obtuvieron mayor puntaje que esa cota, el alza en el puntaje promedio el 2011 es también relevante y alcanza a 5 puntos.

El efecto que sugiere la introducción de la BVP en los puntajes se aprecia en la Figura $\mathrm{N}^{\circ} 1$, que muestra la composición de alumnos seleccionados en Educación por puntaje. Entre 2007 y 2010, período en el que no hubo cambio de condiciones de acceso, la composición se mantuvo, mientras que el año 2011 aumentó de manera notoria la cantidad de alumnos sobre 600 puntos promedio, y naturalmente, al haberse sometido todas las universidades de la muestra a los requeri- 
mientos de la BVP, no existen alumnos seleccionados con menos de 500 puntos. Notoria aparece la moda de la distribución en torno a los 600 puntos y el aumento de la masa de postulantes con más de 600 puntos, elegibles para la BVP, el que se incrementó en $41 \%$ respecto del año anterior.

En cuanto a las características sociodemográficas, la Tabla $\mathrm{N}^{\circ} 2$ muestra que el año 2009 los alumnos seleccionados eran preferentemente mujeres, de ingreso familiar bajo e incluso menor que el de la media de los inscritos. También muestra que no exhiben un perfil claro respecto del tipo de escuela de origen. Ellos presentaban bajos puntajes en la PSU, eran relativamente buenos alumnos según el ranking de resultados del SIMCE respecto de los estudiantes inscritos en la PSU, pero algo inferiores que la media de los seleccionados en las distintas carreras.

FIGURA $N^{\circ}$ 1: $\quad$ DISTRIBUCIÓN DE PUNTAJE PROMEDIO PSU PARA POSTULANTES SELECCIONADOS EN EDUCACIÓN

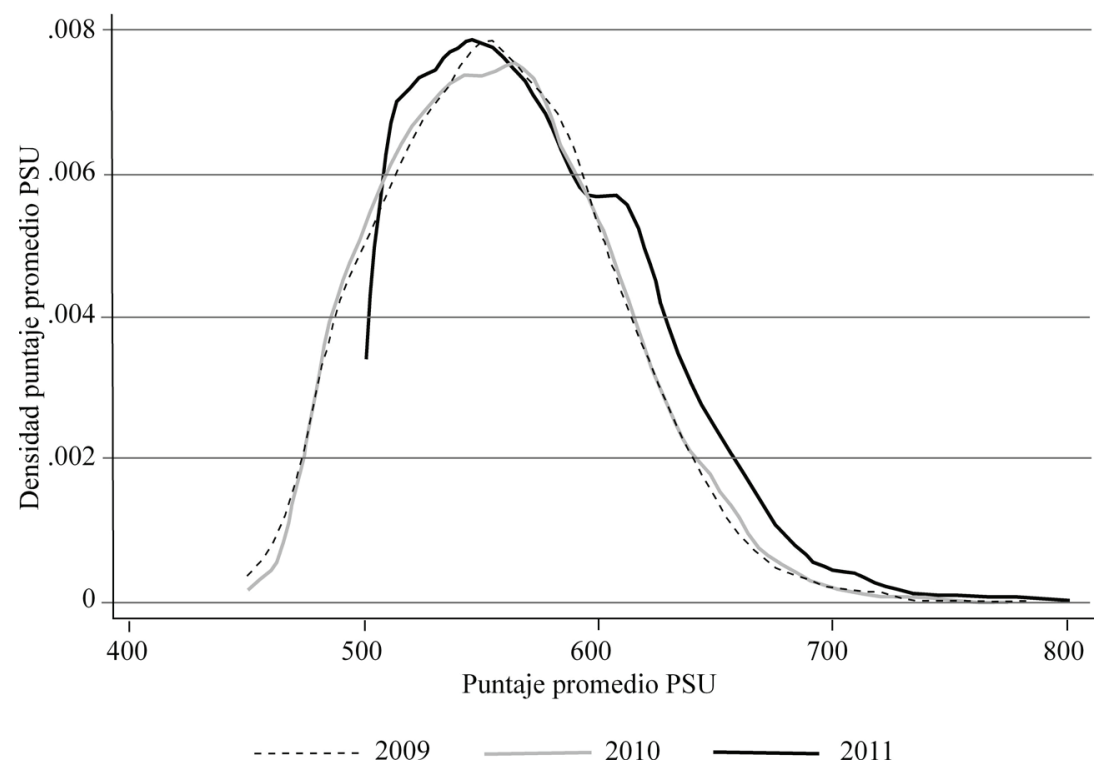

Fuente: Elaboración propia en base Archivos PSU-DEMRE 2009-2011. 
TABLA N 2: CARACTERÍSTICAS DE SELECCIONADOS EN CARRERAS DE EDUCACIÓN DEL CRUCH, DEL TOTAL DE SELECCIONADOS EN CUALQUIER CARRERA Y DEL TOTAL DE INSCRITOS EN LA PSU PARA EL PROCESO DE ADMISIÓN 2009

\begin{tabular}{lrcc}
\hline Variable & $\begin{array}{c}\text { Media de } \\
\text { seleccionados } \\
\text { Educación }\end{array}$ & $\begin{array}{c}\text { Media de } \\
\text { seleccionados } \\
\text { totales }\end{array}$ & $\begin{array}{c}\text { Media de } \\
\text { inscritos } \\
\text { PSU }\end{array}$ \\
\hline Mujer & $71,80 \%$ & $47,50 \%$ & $54,40 \%$ \\
Ingreso familiar (miles de & 510.6 & 823.7 & 568.7 \\
pesos 2013) & $44,1 \%$ & $33,3 \%$ & $41,1 \%$ \\
Escuela municipal & $50,3 \%$ & $48,5 \%$ & $48,4 \%$ \\
Escuela part. subven. & $5,6 \%$ & $16,5 \%$ & $10,7 \%$ \\
Escuela particular & 556,8 & 600,4 & 500,4 \\
Puntaje PSU & $32,6 \%$ & $29,3 \%$ & $45,9 \%$ \\
Ranking SIMCE & & & \\
\hline
\end{tabular}

FIGURA N ${ }^{\circ}$ 2: $\quad$ CAMBIO PORCENTUAL DE SELECCIONADOS EN PEDAGOGÍA CON MÁS DE 600 PUNTOS RESPECTO DEL AÑO ANTERIOR POR GÉNERO (UNIVERSIDADES DEL CRUCH).

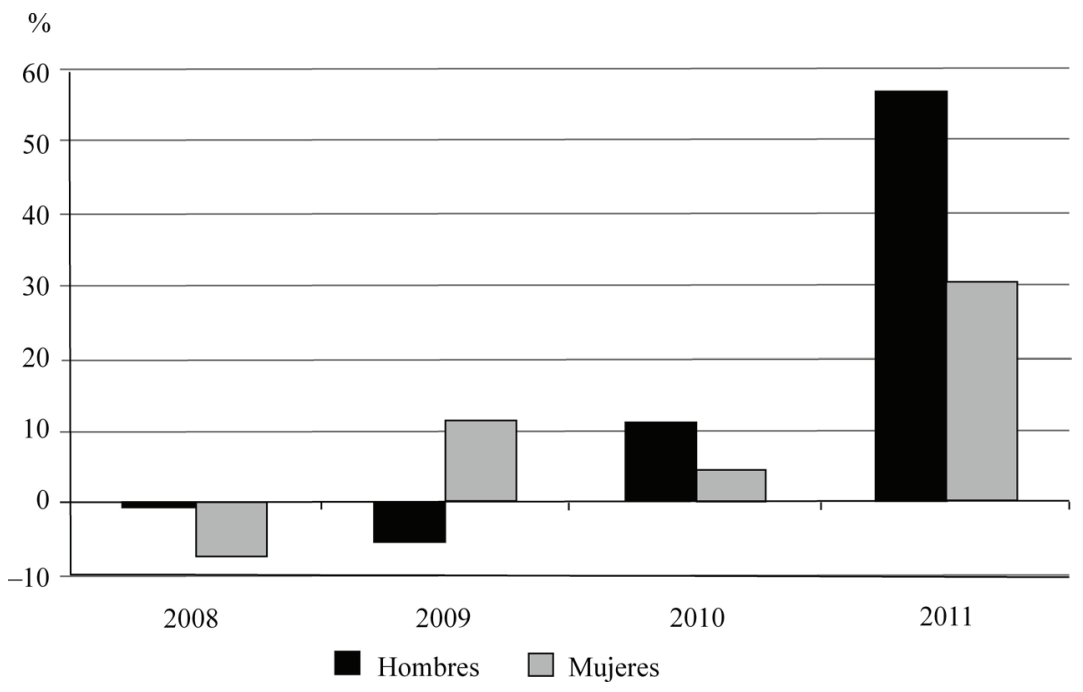


FIGURA N ${ }^{\circ}$ 3: CAMBIO PORCENTUAL, RESPECTO AL AÑO ANTERIOR, DE ADMITIDOS EN PEDAGOGÍA CON MÁS DE 600 PUNTOS PSU, POR TIPO DE ESTABLECIMIENTO (universidades del CRUCh)

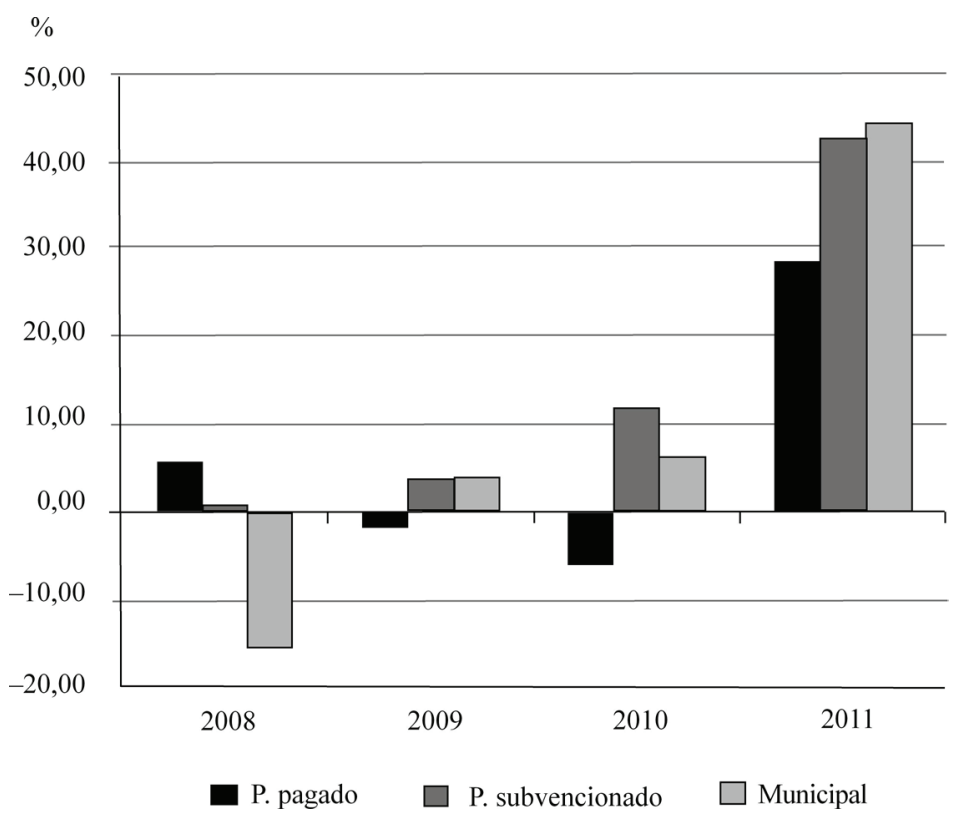

Las Figuras $N^{\text {os. }} 2$ y 3 muestran los cambios porcentuales de alumnos con más de 600 puntos en la PSU, por género y tipo de establecimiento, respectivamente. La BVP está asociada a un incremento en el porcentaje de hombres y a un aumento de los seleccionados provenientes de la educación pública (sector municipal).

\section{Impacto de la BVP}

Lo señalado en la sección anterior muestra una clara relación entre la BVP y el mejoramiento en los puntajes de ingreso a las carreras de Educación, lo que viene a ratificar el trabajo con el método de discontinuity regressions realizado por Alvarado et al. (2012). En esta sección cuantificamos la magnitud del efecto y procuramos distinguir cuánto de éste obedece a una recomposición de postulantes hacia alum-

${ }^{5}$ Cabe señalar que en Chile la educación municipal concentra mayor porcentaje de alumnos vulnerables y una sustancial mayor varianza en los aprendizajes, respecto de la educación particular. Para una revisión, véanse Drago y Paredes (2011) y las referencias a distintos trabajos respecto de Chile. 
nos de mayor ingreso y cuánto se debe a un efecto de recomposición de los alumnos con otros factores no medidos que pudiéramos asociar, al menos en parte, a habilidades.

\subsection{Alumnos atraídos por la BVP}

Para determinar las características del tipo de alumnos atraídos por la BVP, identificamos los factores que se correlacionan con la elección de carrera y el perfil del alumno aceptado a pedagogía el año 2009, contrastándolo con el perfil del estudiante de Educación del 2011. Utilizamos las bases de datos de los postulantes a universidades del CRUCh provistas por el DEMRE para dichos años, además de los datos del SIMCE de segundo año medio por alumno ${ }^{6}$. Primeramente estimamos el efecto de dichos factores en la probabilidad que un alumno postulara y fuera aceptado en pedagogía para los años 2009 y el 2011, usando la especificación (1). Dado que queremos obtener el efecto de cada variable sobre haber sido seleccionado, una variable binaria, optamos por una estimación Probit.

$E(y \mid X)=\operatorname{Prob}(y=1 \mid X)=F(X)=F($ mujer,edmadre,NEM,PSU,Rank Simce, dependencia,ingreso)

En este modelo $y$ es una variable binaria que toma el valor 1 si el postulante fue seleccionado en Educación en el proceso respectivo (2009 o 2011), y 0 si fue seleccionado en un área diferente. Las variables de control incluidas en $X$ comprenden factores demográficos, socioeconómicos y de rendimiento del estudiante: Mujer corresponde a una variable binaria que toma el valor 1 si es que el postulante es mujer y 0 en el caso contrario, edmadre reporta los años de educación de la madre, $N E M$ corresponde al promedio de notas de enseñanza media (con rango 10 a 70), PSU corresponde al puntaje promedio PSU en Matemática y Lenguaje, RankSimce corresponde al lugar porcentual del alumno de acuerdo a su puntaje promedio en SIMCE en segundo medio referido a otros alumnos del mismo nivel en su misma escuela, depen-

${ }^{6}$ Sólo se consideran aquellos alumnos que rindieron la PSU el 2009 y el 2011 y, además, rindieron el SIMCE en segundo medio el año 2006 y 2009 . 
dencia caracteriza el tipo de escuela a la que asistió el alumno (municipal, privada subvencionada, y particular pagada, tomando esta última como categoría base) e ingreso, que corresponde al ingreso familiar. Además, para cada una de las variables de control se incluye un factor de interacción con una variable binaria $d 2011$ que toma el valor 1 si es que el estudiante pertenece al proceso de selección 2011 o 0 en el caso contrario. Los resultados de las estimaciones de (1) realizadas mediante un Probit y expresados en coeficientes marginales, se presentan en la Tabla $\mathrm{N}^{\circ} 3$.

TABLA N ${ }^{\circ}$ 3: $\quad$ EFECTOS MARGINALES EN LA PROBABILIDAD DE SER SELECCIONADO EN PEDAGOGÍA EN UNIVERSIDADES DEL CRUCH

\begin{tabular}{|c|c|c|}
\hline Variables & 2009 & Diferencia 2009-2011 ${ }^{\mathrm{a}}$ \\
\hline Mujer & $\begin{array}{c}0.09596 * * * \\
(0.00259)\end{array}$ & $\begin{array}{l}-0.0032 \\
(0.0042)\end{array}$ \\
\hline Educación madre (años) & $\begin{array}{c}-0.0026^{* * * *} \\
(0.00041)\end{array}$ & $\begin{array}{c}-0.00172 * * \\
(0.0007)\end{array}$ \\
\hline Ingreso (en \$10.000) & $\begin{array}{c}-0.00028 * * * \\
(0.00003)\end{array}$ & $\begin{array}{l}-0.00009^{*} \\
(0.000051)\end{array}$ \\
\hline Municipal & $\begin{array}{c}0.01479 * * * \\
(0.0056)\end{array}$ & $\begin{array}{l}-0.0141^{*} \\
(0.00794)\end{array}$ \\
\hline Subvencionado & $\begin{array}{c}0.0078 \\
(0.0049)\end{array}$ & $\begin{array}{c}-0.0108 \\
(0.00757)\end{array}$ \\
\hline Notas enseñanza media (décimas) & $\begin{array}{c}-0.00473 * * * \\
(0.00031)\end{array}$ & $\begin{array}{c}-0.00386 * * * \\
(0.00039)\end{array}$ \\
\hline Puntaje PSU & $\begin{array}{c}-0.00087^{* * *} \\
(0.000024)\end{array}$ & $\begin{array}{c}0.000467 * * * \\
(0.000038)\end{array}$ \\
\hline Ranking SIMCE & $\begin{array}{l}-0.00012 * * \\
(0.000054)\end{array}$ & $\begin{array}{l}0.00024 * * * \\
(0.000082)\end{array}$ \\
\hline $\begin{array}{l}\text { Pseudo } \mathrm{R}^{2} \\
\text { Observaciones }\end{array}$ & \multicolumn{2}{|c|}{0.145} \\
\hline
\end{tabular}

Notas:

Efectos calculados en el valor promedio de cada variable.

Errores estándar entre paréntesis.

p: nivel de significancia $* * * \mathrm{p}<0.01, * * \mathrm{p}<0.05,{ }^{*} \mathrm{p}<0.1$.

${ }^{\text {a }}$ La diferencia entre el 2009 y 2011 se considera como el efecto de la variable interactuada con la variable binaria $d 2011$, que toma el valor 1 si es que el estudiante pertenece al proceso 2011 y 0 en caso contrario. 
Las diferencias en las características de los estudiantes se observan en los cambios de los coeficientes entre los años 2009 y 2011 (tercera columna de Tabla $\mathrm{N}^{\circ} 3$ ). La mayor parte de las variables tiene un cambio significativo entre los años 2009 y 2011, a excepción del efecto de ser mujer o pertenecer a un colegio subvencionado. A modo de ejemplo, un mejor ranking en el SIMCE se relaciona con menor probabilidad de ser seleccionado en una carrera de pedagogía, pero ello ocurre con menor fuerza en el año 2011. Si, por el contrario, nada hubiera pasado, excepto un aumento en la probabilidad de que todos los estudiantes, independientemente de su perfil, postularan y fueran aceptados, entonces los coeficientes serían los mismos y la distribución de características no habría cambiado. Sabemos, sin embargo, que el hecho de que aumentara el puntaje de corte a 500 puntos modificó el tipo de estudiantes elegibles, pero es posible que también la misma BVP haya afectado las características de los estudiantes que tenían mayores puntajes. Para analizar esta hipótesis realizamos el mismo ejercicio entre los años 2006 y 2009 y hallamos que ninguna de las variables entre ambos años observa diferencias significativas, a excepción del efecto de educación de la madre. Sin embargo, la diferencia en magnitud no era sustancialmente relevante.

El efecto del cambio en las probabilidades y del tipo de estudiantes lo podemos computar a partir de los parámetros reportados en la Tabla $\mathrm{N}^{\circ} 3$ que permiten, para estudiantes con determinadas características, obtener un predictor de la probabilidad de haber sido seleccionado en pedagogía. En base a las probabilidades predichas para cada año para cada estudiante $\left(\hat{e}_{i}^{y}\right)$, obtenemos la diferencia de probabilidades $\alpha_{i}$ que un mismo postulante $i$ fuera seleccionado en pedagogía si hubiese postulado el año 2009 y el 2011.

$$
\alpha_{i}=\hat{e}_{i}^{11}-\hat{e}_{i}^{09}
$$

Los valores de $\alpha_{i}$ tienen una media de -0.014 y una desviación estándar de 0.038 . Una diferencia $\alpha_{i}$ mayor que cero puede interpretarse como que para un postulante con determinadas características la BVP hizo más atractiva la carrera de Educación, puesto que su aparición aumentó la probabilidad de elegir esa carrera. 
Para determinar el efecto del cambio en la composición, vinculado al cambio de probabilidades de postular y ser seleccionado según características, reponderamos la muestra observada el año 2009 por el factor $\mathrm{F}_{\mathrm{i}}=\left(1+\alpha_{i}\right)$, asociable a la diferencia de probabilidades que introduce la BVP. La muestra del 2009 reponderada puede interpretarse como aquella que refleja exclusivamente el efecto en el tipo de estudiantes que son inducidos por la BVP a estudiar pedagogía, ya que asigna un mayor peso proporcional a aquellas observaciones que aumentan su probabilidad de postular a Educación entre los años 2009 y 2011, inducidos por la BVP, y asigna un menor peso a aquellos postulantes para los que su probabilidad de postulación disminuye entre ambos procesos. Consecuentemente, las diferencias de las medias de las características de la muestra reponderada y de la original muestran el cambio en el perfil de estudiantes inducidos por la BVP, ya que, usando la misma muestra (postulantes 2009), se captura el efecto de la beca en el cambio de las probabilidades de postulación.

La Tabla $\mathrm{N}^{\circ} 4$ caracteriza en su primera columna a los estudiantes bajo 500 puntos que fueron sacados del sistema por la BVP el 2011. Las columnas siguientes muestran las características promedio de los postulantes a Educación seleccionados el 2009 y el 2011 en rangos de puntaje PSU, y las características de los seleccionados el 2009 con más de 500 puntos PSU corregidas por sus respectivas ponderaciones $\mathrm{F}_{\mathrm{i}}$. Ello representa el efecto de la BVP sobre la composición de los estudiantes si ésta hubiese sido aplicada sobre los postulantes del 2009. Debido a que tanto la distribución como las características de los estudiantes seleccionados en carreras de Educación entre los años 2006 y 2010 no tienen diferencias sustantivas, se puede asumir que el cambio entre el proceso 2009 y 2011 se debe principalmente al efecto de la introducción de la BVP.

También se observa que los seleccionados el 2009 que obtuvieron bajo 500 puntos provienen de estratos socioeconómicos más vulnerables y de peor ranking dentro de su generación. Sorprende el hecho de que los postulantes 2011 con puntajes mayores que 600 puntos PSU promedio tienen menor ingreso familiar y mejor rendimiento en el SIMCE que los del 2009. Esto sugiere que el aumento de puntajes PSU no se debe a que los aceptados son estudiantes de mejor situación socioeconómica, sino a que son estudiantes de mejor rendimiento. 
TABLA N ${ }^{\circ}$ :

CARACTERÍSTICAS DE SELECCIONADOS EN EDUCACIÓN SEGÚN SU PROMEDIO PSU

\begin{tabular}{|c|c|c|c|c|c|c|c|}
\hline \multirow[t]{2}{*}{ Variables } & \multicolumn{3}{|c|}{2009} & \multicolumn{2}{|c|}{2011} & \multicolumn{2}{|c|}{$\begin{array}{c}\text { Postulantes } 2009 \\
\text { ponderados usando } F^{i}\end{array}$} \\
\hline & $<500$ & $500-600$ & $>600$ & $500-600$ & $>600$ & $500-600$ & $>600$ \\
\hline Es mujer & $80,4 \%$ & $71,40 \%$ & $67,2 \%$ & $70,7 \%$ & $60,8 \%$ & $71,01 \%$ & $67,8 \%$ \\
\hline $\begin{array}{l}\text { Ingreso (en miles } \\
\text { de pesos) }\end{array}$ & 331.9 & 412.0 & 820.0 & 375.8 & 712.8 & 411.2 & 792.7 \\
\hline $\begin{array}{l}\text { Puntaje PSU } \\
\text { promedio }\end{array}$ & 484.1 & 550.4 & 629.9 & 548.2 & 635.3 & 550.8 & 630.1 \\
\hline $\begin{array}{l}\text { Educación madre } \\
\text { (en años) }\end{array}$ & 11.0 & 12.0 & 13.6 & 11.8 & 13.4 & 11.9 & 13.5 \\
\hline $\begin{array}{l}\text { Educación padre } \\
\text { (en años) }\end{array}$ & 10.9 & 12.0 & 13.7 & 11.8 & 13.7 & 12.0 & 13.7 \\
\hline Ranking SIMCE* & $39,11 \%$ & $32,65 \%$ & $28,11 \%$ & $34,18 \%$ & $26,8 \%$ & $32,49 \%$ & $28,9 \%$ \\
\hline Municipal & $59 \%$ & $45 \%$ & $32 \%$ & $44 \%$ & $33 \%$ & $41 \%$ & $30 \%$ \\
\hline Subvencionado & $41 \%$ & $52 \%$ & $50 \%$ & $53 \%$ & $53 \%$ & $55 \%$ & $50 \%$ \\
\hline Particular & $0 \%$ & $3 \%$ & $18 \%$ & $3 \%$ & $14 \%$ & $4 \%$ & $20 \%$ \\
\hline
\end{tabular}

* El ranking SIMCE corresponde al lugar (porcentual) del estudiante en el SIMCE con respecto a sus compañeros de generación del mismo establecimiento.

Para determinar más claramente el efecto previo, separamos la muestra de postulantes en base al cambio de probabilidades $\alpha_{i}$. La Tabla $\mathrm{N}^{\circ} 5$ divide la muestra en dos grupos: el $25 \%$ de menor probabilidad (los que, dadas sus características, tienen menos probabilidades de que serán seleccionados en pedagogía el 2011), y el 25\% superior (los que, dada la BVP, aumentan de manera notoria su probabilidad de quedar seleccionados en Educación entre el 2009 y el 2011). Se eligen las dos colas de la distribución para analizar las diferencias entre los grupos que más probablemente serían afectados por la BVP. Se puede observar que los postulantes que tienen menor probabilidad de estudiar Educación el 2011 con respecto al grupo 2009 son mayoritariamente mujeres, de menor ingreso socioeconómico y menor puntaje PSU. Por el contrario, los postulantes que se encuentran en el $25 \%$ superior de la distribución provienen de estratos socioeconómicos más altos y obtuvieron un promedio PSU también más alto, además de tener una mejor preparación según su ranking SIMCE. 
TABLA N ${ }^{\circ}$ 5: CARACTERÍSTICAS DE POSTULANTES SELECCIONADOS EN EDUCACIÓN EN UNIVERSIDADES DEL CRUCH EL AÑO 2009 SEGÚN SU DIFERENCIA DE PROBABILIDAD PREDICHA

\begin{tabular}{lcc}
\hline Variables & $\alpha_{i}$ en el 25\% inferior & $\alpha_{i}$ en el 25\% superior \\
\hline Es mujer & $93,31 \%$ & $54,70 \%$ \\
Ingreso (en miles de pesos) & 434.8 & 477.3 \\
Puntaje PSU promedio & 528.57 & 607.60 \\
Educación de la madre (años) & 12.8 & 11.5 \\
Educación del padre (años) & 12.4 & 12.2 \\
Ranking SIMCE & $41,60 \%$ & $21,5 \%$ \\
Municipal & $42 \%$ & $39 \%$ \\
Subvencionado & $54 \%$ & $53 \%$ \\
Particular & $4 \%$ & $8 \%$ \\
\hline
\end{tabular}

\subsection{Efecto sociodemográfico y "habilidad"}

Una pregunta que sigue naturalmente a esta descripción es si el mayor puntaje promedio que exhiben los postulantes 2011, que es la métrica vinculada a la selección, se debe a un mejor capital socioeconómico asociado a un mayor ingreso familiar y educación de los padres, o bien a una selección de postulantes con características no controlables que reflejan eventualmente mayor habilidad, lo que por cierto no sólo reflejaría dichas habilidades, sino que el conjunto de factores que se relacionarían con una nueva propensión a entrar a la carrera. Específicamente, si producto de la difusión de la BVP son los jóvenes con más información los más probablemente captados, no se tratará de habilidad sino que de estar informado. En igual línea, los residuos reflejan la falta de control sobre acceso a preuniversitario o profesores particulares en la preparación de la prueba de selección.

Distinguir entre el efecto que denominamos habilidad y el efecto de la composición socioeconómica es prácticamente imposible sin mayores datos. Nuestra aproximación es, como se señaló, imperfecta y la hacemos aislando lo que podemos atribuir del resultado de la prueba de selección universitaria, a la explicación que dan los factores sociodemográficos. Ello se hizo a través de quantile regression sobre el puntaje PSU de cada seleccionado a pedagogía el 2011, controlando por las variables socioeconómicas, como en la ecuación (3). Se utiliza 
una quantile regression para minimizar la suma de los errores en torno a mediana, dada la asimetría que pueden presentar las distribuciones ${ }^{7}$. Los resultados de esta regresión se presentan en la Tabla $\mathrm{N}^{\circ} 6$.

$$
\begin{aligned}
& \left.P S U=\alpha_{0}+\alpha_{1} \text { mujer }+\alpha_{1} \text { ln(ingreso }\right)+\alpha_{2} \text { dependencia }+\alpha_{2} \text { RankSimce } \\
& +\alpha_{4} N E M+\alpha_{5} \text { EdMadre }+\alpha_{6} \text { EdPadre }+\alpha_{7} \text { SimceColegio }+\mu
\end{aligned}
$$
TABLA N ${ }^{\circ}$ 6: VARIABLES DETERMINANTES DEL PROMEDIO PSU PARA POSTU- LANTES SELECCIONADOS EN EDUCACIÓN EN UNIVERSIDADES DEL CRUCH EL AÑO 2011

\begin{tabular}{lc}
\hline Variables & Regresión lineal puntaje PSU \\
\hline Es mujer & $-12.439^{* * *}$ \\
& $(1.469)$ \\
ln (ingreso) & $5.412^{* * *}$ \\
& $(1.077)$ \\
Escuela municipal & $-14.624^{* * *}$ \\
& $(3.404)$ \\
Escuela part. subvencionada & $-19.289^{* * *}$ \\
& $(2.098)$ \\
Ranking SIMCE & $-0.866^{* * *}$ \\
NEM & $(0.033)$ \\
Educación de la madre (años) & $0.130^{* * *}$ \\
Educación del padre (años) & $(0.009)$ \\
Promedio SIMCE colegio & $1.043^{* * *}$ \\
Constante & $(0.265)$ \\
Observaciones & $0.472^{*}$ \\
& $(0.261)$ \\
& \\
&
\end{tabular}

Errores estándar entre paréntesis; $\mathrm{p}$ : nivel de significancia. $* * * \mathrm{p}<0.01, * * \mathrm{p}<0.05, * \mathrm{p}<0.1$.

${ }^{7}$ Para este caso, sin embargo, la diferencia de efectos entre una regresión OLS y la quantile regression no es relevante. 
De los resultados presentados en la Tabla $\mathrm{N}^{\circ} 6$ obtenemos los residuos, que denominamos habilidades de los postulantes, y que, como señalamos, se asocian a diferencias en el puntaje PSU que no están explicados por factores socioeconómicos ni por el rendimiento escolar previo.

Los resultados sugieren que el residuo para el grupo de jóvenes más probablemente inducidos a elegir Educación como consecuencia de la BVP $\left(\alpha_{i}>0\right)$ es sustancialmente mayor (33 puntos) que el del conjunto de los postulantes a pedagogía el 2011 (Figura $\mathrm{N}^{\circ}$ 4), donde más del $90 \%$ de los postulantes BVP tiene residuos positivos. Esto es, los postulantes inducidos por la BVP parecen ser especialmente "hábiles".

\subsection{Efecto diferenciado por universidad}

Un efecto posible de la BVP es que, por su diseño, ella favorezca relativamente a universidades de mayor reputación y calidad. Ello en primer lugar porque las universidades mejores y más selectivas debie-

FIGURA N ${ }^{\circ}$ 4: $\quad$ DISTRIBUCIÓN DE RESIDUOS DE PUNTAJE PSU PARA POSTULANTES SELECCIONADOS EN EDUCACIÓN EN UNIVERSIDADES DEL CRUCH EL AÑO 2011 Y PARA AQUELLOS INDUCIDOS POR LA BVP

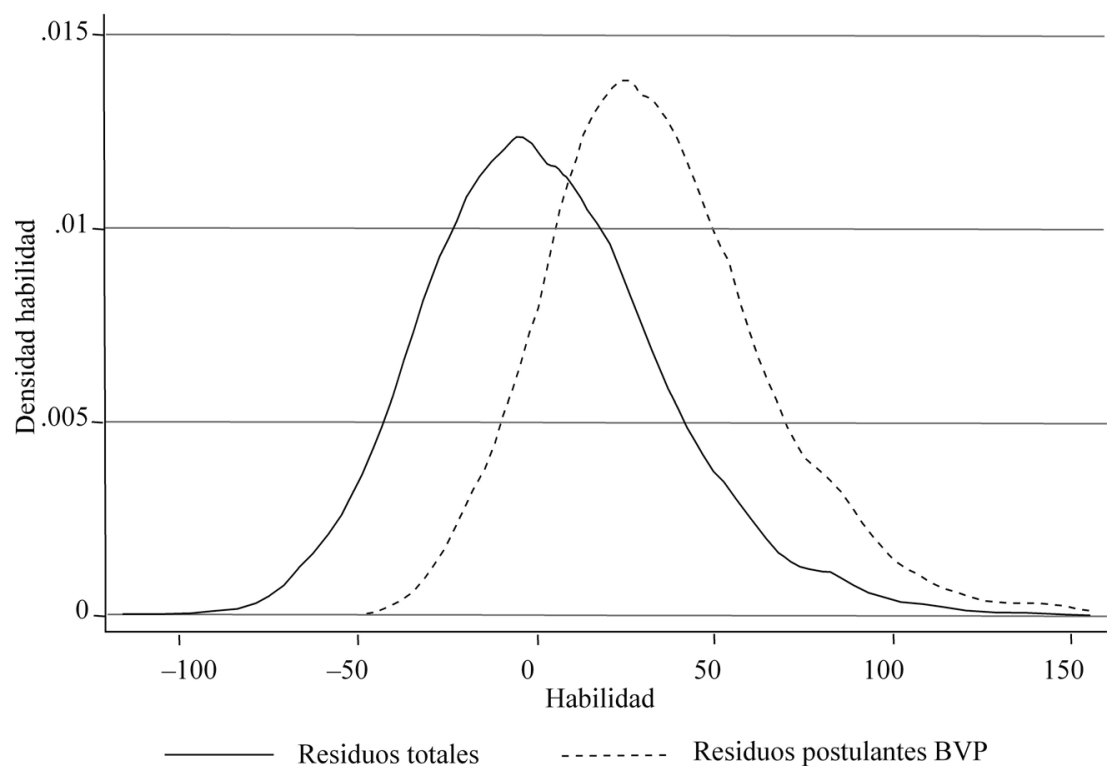


ran tener menos postulantes con puntajes bajo el umbral de 600 puntos. Por su parte, porque hasta la implementación de la BVP, un alumno eventualmente elegible a una universidad de mayor calidad pudo haber optado por una de menor calidad debido al mayor costo presumible de estudiar en la primera. Con la BVP, un postulante elegible puede ver reducido el pago a cero, lo que hace que sólo prime en su decisión lo que él o ella consideran una institución de calidad.

Desgraciadamente, los datos no permiten sino tener una aproximación gruesa a esta relevante pregunta. Desde luego, los criterios para diferenciar calidad por universidad son ciertamente discutibles. Un ejercicio muy grueso permite sugerir que en las 22 universidades del CRUCH se aprecia un efecto diferenciado en relación con dos métricas que bajo ciertas condiciones pudieran asociarse a calidad: el valor del arancel y la proporción de alumnos dentro de la universidad que, previo al proceso 2011, habían conseguido sobre 600 puntos en la $\mathrm{PSU}^{8}$.

Para determinar si efectivamente las 22 universidades del $\mathrm{CRUCH}$ que ofrecen carreras de pedagogía se vieron afectadas en forma diferente por la BVP, estimamos una regresión en la que procuramos explicar la cantidad de alumnos atraídos por la BVP que terminaron seleccionados en la institución $i$, en función del valor del arancel de pedagogía en la institución $i$ y de la cantidad de alumnos sobre 600 puntos que tenía esa institución.

$$
\% \text { BVP }=\beta_{0}+\beta_{1} \text { arancel }_{2009}+\beta_{2}{\text { sobre } 600 \text { ptos }_{2009}}+\mu
$$

Se consideró como proxy del mayor atractivo de la BVP $(\%$ BVP) la diferencia entre el año 2009 y el 2011 en la cantidad de postulantes sobre 600 puntos PSU que tenía la universidad, ponderando las observaciones según la cantidad de postulantes admitidos el año 2011, para controlar por las diferencias en tamaños relativos de las instituciones.

${ }^{8}$ Sin necesidad de concordar en que el valor del arancel es una buena proxy de calidad, para las universidades que cobraban más la BVP representa un estímulo relativamente mayor que para las universidades que cobraban menos, ya que la BVP significa cero costo directo. 
TABLA N ${ }^{\circ}$ 7: POSTULANTES INDUCIDOS POR LA BVP EN UNIVERSIDADES DEL CRUCH (DIFERENCIA ENTRE 2009 Y 2011)

\begin{tabular}{lc}
\hline Variables & $N^{\circ}$ de alumnos inducidos BVP \\
\hline & \\
Postulantes sobre 600 el 2009 & $0.175^{* * *}$ \\
& $(0.00061)$ \\
Arancel 2009 (\$10.000 del 2009) & $0.0746^{* * *}$ \\
& $(0.00332)$ \\
Constante & $-14.364^{* * *}$ \\
& $(0.5164)$ \\
Observaciones & 22 \\
$\mathrm{R}^{2}$ & 0.8961 \\
\hline
\end{tabular}

Errores estándar entre paréntesis.

$* * * \mathrm{p}<0.01, * * \mathrm{p}<0.05, * \mathrm{p}<0.1$.

Si la distribución de puntajes en pedagogía entre 2009 y 2011 hubiera permanecido estable, como de hecho permaneció entre 2007 y 2010 (Figura $\mathrm{N}^{\circ}$ 1), un cambio de dicha distribución podría interpretarse como consecuencia sólo de la BVP.

Los resultados de este ejercicio que aparecen en la Tabla $\mathrm{N}^{\circ} 7$ son consistentes con la idea de que los alumnos inducidos por la BVP se concentraron preferentemente en las universidades que previamente eran preferidas por los alumnos con mayor puntaje en la PSU y que, además, existe una relación positiva entre el arancel cobrado por las universidades el 2009 y la cantidad de alumnos que obtuvieron la BVP.

\section{Conclusiones}

En este trabajo hemos analizado el efecto de la BVP instituida por el Estado chileno el año 2010 como un incentivo dirigido a mejorar el perfil académico de los postulantes a las carreras de pedagogía. El principal hallazgo es que el diseño de esta beca logra su objetivo. Si bien sólo se realiza el análisis en universidades pertenecientes al Consejo de Rectores y no en todas las universidades que ofrecen la carrera de pedagogía, los resultados entregan evidencia relevante correspondiente a la BVP para el conjunto del sistema. 
El hecho que la BVP no se restrinja a postulantes provenientes de hogares de bajos ingresos, que premie el puntaje obtenido en la PSU, y que ambas variables, nivel socioeconómico y puntaje, estén positivamente relacionadas, indica que la BVP no está orientada a incidir en la composición socioeconómica de los beneficiarios de la beca, en el sentido de favorecer a los postulantes de menos recursos. Y si bien hallamos un efecto de inducción de alumnos provenientes de hogares de mayores ingresos, por otro lado también encontramos un efecto adicional que no tiene que ver con ingreso y que podemos relacionarlo con habilidades innatas.

Un ejercicio grueso comparativo entre universidades sugiere que las instituciones que ya contaban con alumnos más selectos y que cobraban aranceles más altos reciben una mayor proporción de alumnos beneficiados por la BVP, lo cual entraña un desafío de superación para aquellos planteles que no han sido capaces de atraer postulantes con mejores estándares académicos.

Por último, cabe señalar que aun cuando la existencia de un impacto significativo en el perfil de los que ingresan a estudiar pedagogía es un primer paso, no es aún suficiente para lograr mayor calidad y equidad en la educación escolar. Ello requiere, además, que los mejores profesores no abandonen la profesión y que, en particular, ejerzan también en las escuelas en que se educan los niños más vulnerables, algo que representa un desafío mayor.

\section{REFERENCIAS}

Alvarado, M., F. Duarte y C. Neilson (2012). "Efectos Beca Vocación de Profesor". Documento del Centro de Estudios del Ministerio de Educación. http://www.mineduc.cl/usuarios/mineduc/doc/20120111 1603490.EstudioEfectospreliminaresBecaVocaciOndeProfesor.pdf.

Barber, M. y M. Mourshed (2008). "Cómo Hicieron los Sistemas Educativos con Mejor Desempeño en el Mundo para Alcanzar sus Objetivos". PREAL, $\mathrm{N}^{\circ}$ 41, julio.

Cabezas, V. y F. Claro (2011). "Valoración Social del Profesor en Chile: ¿Cómo Atraer a Alumnos Talentosos a Estudiar Pedagogía?". Temas de la Agencia Pública, Año 6, $\mathrm{N}^{\circ}$ 42, Centro de Políticas Públicas Pontificia Universidad Católica de Chile.

Cabezas, V., F. Gallegos, V Santelices y M. Zarhi (2011). "Factores Correlacionados con las Trayectorias Laborales de Docentes en Chile, con Especial Énfasis en sus Atributos Académicos”. Documento de 
Trabajo, Facultad de Educación, Pontificia Universidad Católica de Chile.

Cabezas, V., F. Bogolasky, R. Paredes, R. Rivero y M. Zahri (2013). "Los Determinantes del Primer Trabajo para Profesores de Educación Básica en la Región Metropolitana". Proyecto FONIDE N F611105.

Comisión de Financiamiento Estudiantil para la Educación Superior (2012). "Análisis y Recomendaciones para el Financiamiento del Sistema de Financiamiento Estudiantil". Documento del Ministerio del Trabajo, marzo. http://www.mineduc.cl/usuarios/mineduc/doc/201203291032 500.InformeComisionAyudaEstudiantilmarzo2012.pdf

Drago, J. y R. Paredes (2011). “The Quality Gap in Chile's Education System”. Cepal Review, 104.

Larrondo, T, C. Figueroa, M. Lara, A. Caro, J. Rojas, y C. Gajardo (2007). "Desarrollo de Habilidades Básicas de Lenguaje y Matemáticas en Egresados de Pedagogía. Un Estudio Comparativo". Universidad de Playa Ancha de Ciencias de la Educación, Valparaíso, Chile.

Ministerio de Educación (2010). Decreto 337, 16 de noviembre de 2010, que reglamenta el programa de becas de educación superior. Recuperado el 12 de agosto de 2012 de http://www.leychile.cl/Navegar?idNorma=10 19588\&idVersion=2010-11-16.

- (2012). Información sobre la Beca Formación de Profesor. http:// www.becavocaciondeprofesor.cl/

Meckes, L. y M. Bascopé (2010). "Caracterización de las Instituciones Formadoras de Docentes de Educación Básica y Destino Laboral de sus Egresados en Chile". Documento de Trabajo, Centro de Estudios de Políticas y Prácticas en Educación de la Pontificia Universidad Católica (CEPPE-UC).

OECD (2009). "Revisión de Políticas Nacionales de Educación: La Educación Superior en Chile". Informe OCDE, Banco Mundial

Paredes, R. y F. Ramírez (2011). "Selección Adversa en la Educación Chilena”. Documento de Trabajo, Escuela de Ingeniería, Pontificia Universidad Católica de Chile.

Wilson, T (2011). "Impacto de la Beca Vocación de Profesor en las Universidades del Consejo de Rectores". Tesis para optar al grado de Licenciado en Física, Pontificia Universidad Católica de Chile. 